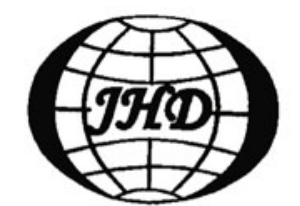

www.sciencedirect.com/ science/journal/10016058

\title{
2017 SPHERIC Beijing International Workshop
}

\section{7-20 October 2017, Beijing}

The 2017 SPHERIC Beijing International Workshop (SPHERIC Beijing 2017) will be held at Peking University(PKU) in Beijing, China during 17-20 October 2017, and this is the first time that the SPHERIC Workshop is held outside Europe.

The SPHERIC workshops are the only worldwide events which exclusively focus on the Smoothed Particle Hydrodynamics(SPH) methodology and related simulation approaches. SPHERIC Beijing 2017 aims to enable experienced researchers and $\mathrm{Ph}$. D. students using SPH to share and contribute to the development and applications of the SPH method.

\section{Workshop Topics:}

- Computational Modelling using SPH

- Theoretical and Numerical Aspects of SPH

- Numerics

- Alternative Formulations and Particle-based Simulation Techniques

- SPH applications

\section{Important dates}

- Abstract Submission Deadline: 20 June 2017

- Announcement of Selected Abstracts:10 July 2017

- Early Registration Deadline:10 August 2017

- Final Paper Submission Deadline:31 August 2017

- Presenter Registration Deadline:10 September 2017

- Training Day:17 October 2017

- Workshop: 18-20 October 2017

\section{Abstract and Paper Submission:}

Abstracts should be one-page long and must include one illustrative figure outlining the main results. Moreover, abstracts are expected to address three major review criteria including (a) novelty, (b) applicability \& impact, and (c) predictive accuracy \& predictive improvements. After notification of accepted abstract, authors are encouraged to prepare the full 8-page paper. Templates of abstract and full paper will be available online.

\section{Conference Venue:}

SPHERIC Beijing 2017 will take place in Peking University (PKU), China. Peking University is the Top University in China with a very beautiful campus. PKU is easily accessible by bus, taxi and subway. Beijing is the capital city of China with direct flights to major cities from all over the world. It hosts many historical relics and tourist attractions like the Forbidden City, the Temple of Heaven, the Summer Palace, the Great Wall and many others.

\section{Sponsor Organizations:}

National Natural Science Foundation of China BIC-EAST, Peking University

Institute of Ocean Research, Peking University

National State Key Laboratory of Turbulence and Complex System, Peking University

\section{Supporting Journals:}

A number of selected papers can be considered to publish on the following journals after peer review:

International Journal of Applied Mechanics International Journal of Computational Methods Journal of Hydrodynamics

\section{Official Website:}

More detailed information on conference venue, abstract and paper submission, registration, travel and accommodation will be available on the workshop website (ocean.pku.edu.cn/SPHERIC_Beijing)

\section{Contact:}

Moubin Liu

Chair of the Local Organizing Committee

Professor, Ph. D.

College of Engineering,

Peking University

Email:mbliu@pku.edu.cn

Tel:+86-10-62766982 